\title{
French Academy of Sciences and the new orthography
}

\section{[L’Academie française et la « nouvelle orthographe »]}

\author{
Michele Lenoble-Pinson
}

DOI: $10.18355 /$ XL.2019.12.01XL.01

\begin{abstract}
Why do learners readily adopt the orthographic corrections from 1990? They eliminate inconsistencies and irregularities (bonhommie aligns with bonhomme). They rationalize the singular and the plural of the compound names of the type portebagage, the conjugation of the verbs in -eler and -eter, the writing of borrowed words (weekend, désidérata) as well as their plural (des matchs, des whiskys). They largely correspond to the natural evolution of pronunciation and spelling.

They touch 2,400 words. The Petit Larousse and the proofreaders take this into account. If we apply them all, less than one word per page is changed. Often, change only affects one accent (allègement, connaitre, couter).

Without necessarily being taught, the new spellings are widely used in Belgium, Quebec, Switzerland and France. Traditional spellings remain valid. Neither of the two spellings (neither the old nor the new) can be held to be at fault. The rectifications from 1990, which are orthographic variants, are recommended by the French Academy of science.
\end{abstract}

Key words: new spelling, rectifications, rationalization, evolution, use, variant, French Academy of science

\section{Résumé}

Pourquoi les apprenants adoptent-ils volontiers les rectifications orthographiques de 1990 ? Elles suppriment des incohérences et des irrégularités (bonhommie s'aligne sur bonhomme). Elles rationalisent le singulier et le pluriel des noms composés du type porte-bagage, la conjugaison des verbes en -eler et -eter, l'écriture des mots empruntés (weekend, désidérata) ainsi que leur pluriel (des matchs, des whiskys). Elles correspondent en grande partie à l'évolution naturelle de la prononciation et des graphies.

Elles touchent 2400 mots. Le Petit Larousse et les correcteurs informatiques en tiennent compte. Si on les applique toutes, moins d'un mot par page est modifié. Souvent, le changement ne touche qu'un accent (allègement, connaitre, couter).

Sans être nécessairement enseignées, les nouvelles graphies se répandent dans l'usage en Belgique, au Québec, en Suisse et en France. Les graphies traditionnelles restent valables. Aucune des deux graphies (ni l'ancienne ni la nouvelle) ne peut être tenue pour fautive. Les rectifications de 1990, qui constituent des variantes orthographiques, sont recommandées par l'Académie française.

Mots-clés : nouvelle orthographe, rectifications, rationalisation, évolution, usage, variante, Académie française

L'orthographe française suscite les passions. Elle est quasi sacrée. Selon certains, l'on ne peut y toucher. Les difficultés orthographiques contribueraient à la beauté de la langue. Pourtant, les irrégularités de la norme orthographique pèsent sur l'apprentissage de l'écrit. Elles donnent du français une image de langue difficile. Dans l'article orthographe du Grand dictionnaire universel du XIX $X^{e}$ siècle (1874), Pierre Larousse constate : " nous passons une grande partie de notre vie à apprendre à

XLinguae, Volume 12 Issue 1XL, January 2019, ISSN 1337-8384, eISSN 2453-711X 
écrire en français, et [...] les plus instruits et les plus intelligents d'entre nous n'y parviennent qu'imparfaitement ». Des voix s'élèvent en faveur d'un allègement ${ }^{l}$.

\section{Peut-on toucher à l'orthographe sans nuire à la langue ?}

Oui, on peut rationaliser l'orthographe sans nuire à la langue parce que l'orthographe n'est pas la langue. Beaucoup les confondent. L'orthographe est le vêtement de la langue. Depuis le $\mathrm{XVI}^{\mathrm{e}}$ siècle, elle a beaucoup changé et elle changera encore. Même si, dans les livres qui paraissent actuellement, les graphies de Montaigne ou de Molière sont modernisées par les éditeurs, les textes de ces auteurs continuent d'appartenir à la langue et à la littérature françaises. L'orthographe des siècles antérieurs a évolué. Le nombre de changements est très élevé.

Si l'on compare une édition du XVII ${ }^{\mathrm{e}}$ siècle avec une édition contemporaine d'une pièce de Molière, on compte une centaine de différences par page : sept, par exemple, dans deux vers de la dernière scène du Misanthrope, que l'on écrivait alors Misantrope, sans $h$. Alceste s'adresse à Célimène :

«Et quoy qu'avec ardeur je veüille vous haïr,

Trouvay-je un Cour en moy tout prest à m'obeïr?»

«Et, quoique avec ardeur je veuille vous haïr,

Trouvé-je un cœur en moi tout prêt à m'obéir ? »

Si l'on suit les rectifications de 1990, seul un accent est à modifier : trouvè$j e$. En effet, la prononciation a évolué. Depuis longtemps, le son é s'est ouvert en è devant une syllabe contenant un $e$ muet (je); mais l'orthographe est lente à se conformer à la phonétique. L'Académie française atteste : père, règle, règne, zèle, etc., en 1740 ; collège, piège, etc., en 1878. Quand il y a inversion du pronom je, les formes verbales aimè-je, puissè-je, dussè-je, etc., ne prenant pas place dans l'ordre alphabétique du Dictionnaire de l'Académie, se sont dérobées aux correcteurs des épreuves des éditions de 1740 et de 1878. L'oubli est réparé en 1990. Voir infra, $\mathrm{n}^{\circ} \mathrm{B} 4$.

De toute façon, Molière reste Molière. Son texte est écrit en français, que l'orthographe de l'édition de son texte soit ancienne ou actuelle. Faire évoluer des graphies, en corriger certaines, rationaliser l'orthographe, ne signifient pas assassiner la langue, ni l'appauvrir, ni opérer un nivèlement par le bas puisque l'on ne touche pas à la langue, on ne touche qu'à son vêtement.

De plus, le français n'est pas une langue morte comme le latin, c'est une langue vivante. Il est donc normal que son usage évolue. Dans chaque édition de son Dictionnaire, l'Académie modifie l'orthographe de nombreux mots : plus de 6000 sur 18000 en 1740. Les modifications provoquent généralement des gémissements. Poëte a perdu sa poésie avec son tréma, soupirait-on en 1878. Pourtant, « ce n'est pas l'y qui donne la grâce aux nymphes » (Apollinaire).

La prononciation, le vocabulaire commun, les terminologies scientifiques et techniques, comme la féminisation des noms de professions, l'emploi de l'adjectif à la place de l'adverbe, évoluent. Les réformes orthographiques participent à cette évolution. Ainsi Les rectifications de l'orthographe, publiées le 6 décembre 1990, dans les Documents administratifs du Journal officiel de la République française, montrent-elles l'évolution de l'usage. Elles portent sur diverses anomalies

\footnotetext{
${ }^{1}$ Le présent article suit les rectifications orthographiques approuvées par l'Académie française en 1990. En plus des exemples, les mots de l'article touchés par les rectifications sont écrits en caractères italiques.
} 
orthographiques, dont nénufar, les noms composés, le trait d'union, le tréma, la transcription de mots étrangers, les accents et le participe passé laissé suivi d'un infinitif.

Ces rectifications, dont beaucoup ont pour elles le simple bon sens, l'Académie française les a approuvées à l'unanimité le 3 mai 1990. Elles ont reçu l'aval d'instances officielles en France, au Québec, en Suisse romande et en Communauté française de Belgique. Le Conseil international de la langue française (CILF, Paris) a suivi l'Académie en les introduisant dès 1991 dans toutes ses publications sans que personne n'y ait trouvé à redire. Les Championnats d'orthographe de Belgique les admettent depuis 1991. André Goosse les signale, depuis 1993, dans Le bon usage de Grevisse, qu'il ne cesse de mettre à jour (2007, 2011, 2016). Depuis la rentrée scolaire de 2016, les rectifications sont enseignées en France et les éditeurs français de manuels, après les éditeurs belges, en tiennent compte.

Il s'agit de propositions ou de variantes. Elles se présentent, d'une part, sous la forme de modifications de graphies particulières (anomalies, soudure de mots composés et de mots étrangers); d'autre part, sous la forme de sept règles d'application générale.

\section{Quels bénéfices les usagers retirent-ils de l'emploi des rectifications?}

A. 1) Les rectifications suppriment des incohérences et des irrégularités qui se sont accumulées au cours des siècles. La plupart résultent d'accidents de l'histoire de la langue. Avant 1990, on comptait plus de 5000 mots répertoriés sous des graphies différentes selon les dictionnaires.

Les rectifications portent sur des familles désaccordées. Charriot trouve enfin sa place parmi les dérivés de char, comme charrue, charrette, charretier, qui s'écrivent avec deux $r$. Chariot, avec un $r$, est une irrégularité sans raison, qui complique inutilement l'orthographe. Désormais, imbécilité s'aligne sur imbécile; bonhommie, sur bonhomme; cahutte, sur hutte; combattif et combattivité, sur combattre ; innommé, sur nommer, d'où il vient. Innomé écrit avec un seul $m$ contient une erreur.

Les rectifications corrigent des anomalies. Dans $\grave{A}$ la recherche $d u$ temps perdu, lorsque Marcel Proust décrit les nénufars de la Vivonne, il écrit le mot avec $f$ : «Bientôt le cours de la Vivonne s'obstrue de plantes d'eau. Il y en a d'abord d'isolées comme tel nénufar ». À l'époque, telle était l'orthographe de l'Académie française, dans la $7^{\mathrm{e}}$ édition (1878) de son Dictionnaire, comme dans les éditions précédentes. Cependant, des étymologistes, ne connaissant pas l'arabe nînufar, ont cru que le mot français venait de nymphea; d'où, la graphie avec $p h$ introduite dans la $8^{\mathrm{e}}$ édition (1932-1935). Or nénufar est emprunté, par l'intermédiaire de l'arabe, au persan nîlûfar. Ni en persan ni en arabe n'existe la graphie $p h$. La seule graphie conforme à l'étymologie est nénufar avec $f$, forme correcte que recommande l'Académie depuis 1990. Pourquoi tant d'oppositions?

Les deux participes passés absous et dissous sont refaits, d'après leur féminin absoute et dissoute, en absout et dissout. Ils étaient les seuls mots français présentant l'alternance masculin en $-s$, féminin en -te.

Dans levraut, le suffixe -aud écrit -aut (forme unique), contient une valeur diminutive exceptionnelle. Aussi la graphie levraut est-elle remplacée par levreau, dont le suffixe -eau sert particulièrement à désigner le petit d'un animal, ce qui rapproche le mot de chevreau, lapereau, renardeau, etc.

Ces aménagements, modérés - il faut le reconnaitre -, tendent vers une plus grande cohérence des graphies françaises. La liste complète des formes modifiées se 
trouve sur le site du Réseau pour la nouvelle orthographe du français (RENOUVO) : http://www.renouvo.org/liste.

2) Les rectifications favorisent la soudure des éléments de mots composés parce qu'elle facilite l'application des marques du pluriel. La soudure, en effet, consiste à écrire en un seul mot, sans trait d'union ni espace, les éléments d'un mot composé, qui, dès lors, se présente comme un mot unique.

Pourquoi souder les éléments de mots composés ? Parce qu'ils ne sont pas ou ne sont plus analysables par l'usager, soit que l'un d'eux ait cessé d'être en usage (port dans passeport), soit que leur association ne soit plus, du point de vue syntaxique ou sémantique, compréhensible aujourd'hui. Une chauvesouris ne s'oppose pas à une souris chevelue. Millepatte peut s'écrire soudé comme millefeuille. Même s'il a beaucoup de pattes, l'insecte appelé millepatte n'en a pas mille, pas plus que la pâtisserie appelée millefeuille ne contient mille feuilles. En composition, mille a perdu son sens ; dès lors, le mot composé peut être soudé, considéré comme un mot unique et fonctionnant comme tel.

De même sont soudés divers noms, ancrés dans l'usage, devenus inanalysables pour l'usager: bassecour, chaussetrappe, croquemitaine, croquemort, fairepart, piquenique, quotepart, sagefemme, terreplein, vanupied, volteface. Leur pluriel, régulier, se marque par un $s$ à la finale du nom: des chaussetrappes, des millepattes.

Se soudent les noms composés, bien implantés dans l'usage, dont le second élément est tout ou partout: brisetout, essuietout, faitout, fourretout, mangetout, mêletout, risquetout, passepartout, vatout; ainsi que les noms composés dont le premier élément est porte-: porteclé, portecrayon, portemine, portemonnaie, portevoix. S'ajoutent tirebouchon et couvrepied. Leur pluriel, régulier, se marque par un $s$ à la finale du nom : des faitouts, des porteclés.

Se soudent les mots composés dont le premier élément est contr(e)- ou entr(e)-. Ils étaient déjà soudés dans bien des cas. Devant une voyelle, le e disparait : contre + attaque $=$ contrattaque. On peut écrire contrechant $($ comme contrechamp $), \grave{a}$ contrecourant (comme à contresens), contrespionnage (comme contrescarpe), contrappel (comme contrordre), contrindiqué, controffensive; s'entraimer (comme s'entraider), entrapercevoir, s'entrappeler, s'entredéchirer, s'entredévorer, $s^{\prime}$ 'entretuer (comme s'entremêler). La règle ne touche pas les mots composés de plusieurs éléments comme entre-deux-guerres ou contre-la-montre, dans lesquels entre et contre ne sont pas de simples préfixes.

Se soudent les mots composés dont le premier élément est extra-, intra-, ultra-, infra-, comme les composés de co-, sur-, supra-, qui sont déjà soudés. On écrit extraconjugal (comme extraordinaire), extraterrestre, intraoculaire, intraveineuse, ultrason, ultraviolet, infrasonore.

Le trait d'union est maintenu dans les mots où la soudure entrainerait une prononciation défectueuse, lorsque le $a$ est suivi de $i$ ou de $u$ (pour éviter ai, ain, $a u$ ): extra-institutionnel, grossesse extra-utérine, vie intra-utérine.

Se soudent les nombreux mots composés d'éléments savants, en particulier en $-o$, déjà très souvent écrits sans trait d'union: aéroclub, agroalimentaire, audiovisuel, autoévaluation, cardiovasculaire, électroménager, microéconomie, minijupe, néogothique, néozélandais, otorhinolaryngologiste (même si l'Académie juge le trait d'union préférable dans les mots formés de plus de deux composantes), radioactif, rhinopharyngite, socioculturel, téléfilm, vidéocassette, etc. 
Le trait d'union est maintenu lorsqu'il sert à marquer une relation de coordination entre deux termes désignant des noms propres ou géographiques : mythe gréco-romain, relations franco-russes, culture finno-ougrienne.

On conserve aussi le trait d'union dans les cas où la soudure entrainerait une prononciation défectueuse, lorsque le $a$ ou le $o$ est suivi de $i$ ou de $u$ (pour éviter ai, ain, au, oi, oin, ou) : bio-industrie, hospitalo-universitaire.

Se soudent également les mots empruntés, bien implantés dans l'usage et n'ayant pas valeur de citations, qu'ils soient d'origine étrangère : baseball, basketball, blackout, bluejean, covergirl, cowboy, fairplay, globetrotteur, handball, hotdog, motocross, ossobuco, pipeline, striptease, volleyball, waterpolo, weekend; ou d'origine latine : apriori (nom), exlibris, exvoto, statuquo. On soude principalement les noms.

Les locutions adverbiales latines, écrites avec espace, même si elles sont francisées (avec des accents), ne sont pas soudées : in extrémis, à priori, à postériori, travailler ex nihilo. Dans les textes, il est d'usage d'écrire en italique les termes non francisés, pour montrer leur caractère étranger par rapport à la langue française.

Se soudent aussi les composés d'origine onomatopéique ou expressive, tels que blabla, bouiboui, froufrou (s'écrivait déjà soudé), grigri, kifkif, mélimélo, pêlemêle, pingpong (nom), prêchiprêcha, tamtam, tohubohu, traintrain, tsétsé.

\section{B. Règles modifiées}

1) Les noms composés avec trait d'union du type porte-avion (verbe + nom) ou après-ski (préposition + nom) forment leur singulier et leur pluriel comme s'ils étaient des noms simples : ils prennent la marque du pluriel seulement quand ils sont au pluriel et seul le second élément prend cette marque. Le verbe ne varie pas. Un porte-avion, deux porte-avions. Un compte-goutte, des compte-gouttes. Un essuiemain, des essuie-mains. Un ou une perce-neige, des perce-neiges. - Un ou une aprèsmidi, des après-midis (l'invariabilité manquait de justification). Un hors-jeu, des horsjeux. Un ou une sans-cœur, des sans-cœurs. Des sans-gênes. Des sans-patries. Des sans-papiers.

Dans un sèche-cheveu et un tire-fesse, cheveu et fesse peuvent correctement s'employer au singulier, avec une valeur collective, pour désigner l'ensemble des cheveux, la chevelure, et les deux fesses. Le personnel d'une société de produits capillaires, comme une esthéticienne en art capillaire, ne travaille pas dans les cheveux, mais travaille dans le cheveu. "Soins $d u$ cheveu » (Petit Robert). "Une jeunesse impersonnelle sauvait la fesse musclée » (Colette, La chatte). De même, on dit et on écrit : travailler dans la photo, dans la chaussure, dans l'automobile, etc.

Selon une vieille règle sans fondement, garde varie en nombre dans les composés qui désignent un être humain et reste invariable pour les choses : des gardes-barrière mais des garde-fous, des gardes-côte(s) (douaniers) mais des gardecôtes (bateaux). Or garde n'est pas un nom dans le premier cas, c'est un verbe, comme dans le second. Dès lors, suivant les rectifications de 1990, il convient d'appliquer la règle générale à tous les composés de garde.

Un ou une garde-barrière, des garde-barrières.

Quelques exceptions se justifient par la présence d'un article singulier ou d'un élément nominal écrit avec une majuscule. Ils empêchent la formation du pluriel : des trompe-la-mort, des trompe-l'œil, des sans-le-sou; des prie-Dieu sont invariables.

2) Trait d'union dans les numéraux composés dont les éléments ne sont pas des noms. Vingt-et-un. Vingt-et-unième. Vingt-deux (inchangé). Cent-un. Cent- 
deux. Deux-cents. Trois-mille-trois-cent-quatre-vingt-dix. La règle ne s'applique pas à million et à milliard, qui sont des noms. Deux millions cent. Toutefois, la règle propre à vingt et à cent multipliés et placés ou non à la fin du numéral reste d'application. Quatre-vingts (inchangé). Quatre-cent-mille.

Vingt et un tiers et vingt-et-un tiers correspondent à des valeurs différentes : $20+1 / 3 ; 21 / 3(=21 \times 1 / 3)$.

Selon la règle traditionnelle, on ne met un trait d'union qu'entre deux numéraux inférieurs l'un et l'autre à cent, quand il n'y a pas de et. Comment justifier la différence entre vingt-deux et cent deux ou entre quatre-vingts et quatre cents? La nouvelle règle est plus cohérente.

3) Le tréma se place sur la voyelle $\boldsymbol{u}$ qui doit être prononcée (et non sur une voyelle muette), dans les adjectifs féminins aigüe, ambigüe, contigüe, exigüe, suraigüe, et dans les noms cigüe, ambigüité, contigüité, exigüité. Une douleur aigüe. Des réponses ambigües.

De façon générale, le tréma interdit que l'on prononce deux lettres en un seul son : maïs. Afin d'éviter des prononciations irrégulières (arguer comme narguer, gageure comme heure), un tréma est ajouté sur le $u$ à l'infinitif et dans les formes conjuguées du verbe argüer ainsi que dans les noms gageüre, mangeüre, rongeüre, vergeüre. Le tréma marque la lettre qui se prononce comme voyelle dans ambigüe, nous argüons, quelle gageüre! ou comme semi-voyelle dans ambigüité.

\section{4) Souvent le changement ne touche qu'un accent grave}

Le è avec accent grave remplace le é avec accent aigu dans quatre cas.

$1^{\circ} \mathrm{Au}$ présent (de l'indicatif, du subjonctif et de l'impératif), au futur et au conditionnel de verbes qui ont un é à l'avant-dernière syllabe de l'infinitif, tels que céder, interpréter, régler, cet é se change en è devant une syllabe contenant un $e$ muet, conformément à la prononciation : je cède (inchangé), cède (inchangé), je cèderai, je cèderais.

Jusqu'en 1990, le changement de é en è ne se faisait que devant une syllabe muette finale, ce qui excluait le futur simple et le conditionnel présent. Or, dans je céderai, conserver l'accent aigu constitue une contradiction inutile. D'ailleurs, les futurs et les conditionnels du type cèderai, cèderais, se rencontrent sous la plume des adversaires des rectifications.

$2^{\circ} \mathrm{Au}$ présent (de l'indicatif, du subjonctif et de l'impératif), au futur et au conditionnel des verbes en -eler et -eter, le $e$ muet de l'avant-dernière syllabe de l'infinitif se change en è devant une syllabe contenant un $e$ muet.

Les verbes en -eler se conjuguent comme peler. Sur le modèle de je pèle, on écrit : je démantèle, je harcèle, je ruissèle. Cette dernière forme graphique était celle des trois premières éditions du Dictionnaire de l'Académie française. Le dérivé en -ment est touché également : le ruissèlement.

Les verbes en -eter se conjuguent comme acheter. Sur le modèle de j'achète, on écrit : je décollète, j'époussète, j'étiquète. Cette dernière forme était celle des $5^{\mathrm{e}}, 6^{\mathrm{e}}$ et $7^{\mathrm{e}}$ éditions du Dictionnaire de l'Académie. Le dérivé en -ment est touché également : le cliquètement.

Désormais, l'accent grave remplace la double consonne qui suivait le $e$ auparavant. Ainsi disparaissent nombre d'hésitations. La régularisation, en effet, touche environ deux-cents verbes, au sujet desquels les dictionnaires et les grammaires se contredisent dans vingt-cinq pour cent des cas.

Deux verbes, bien implantés dans l'usage, appeler et jeter, ainsi que leurs composés, conservent leur conjugaison traditionnelle avec double consonne devant le $e$ muet, parce que les experts ont craint qu'en raison de leur haute fréquence, un 
changement ne trouble trop les habitudes visuelles des lecteurs. J'appelle, il rappellera, ils s'entrappelleront; je jette, il rejetterait, ils projetteraient.

$3^{\circ}$ Conformément à la prononciation, à la finale de la $1^{\text {re }}$ personne du singulier, dans la langue littéraire, on remplace le é par un è quand le pronom je est placé après le verbe : puissè-je, dussè-je, eussè-je, fussè-je, trouvè-je (voir supra: Molière), etc.

$4^{\circ}$ Dans les mots pour lesquels l'usage hésitait entre les graphies é ou $\grave{e}$, on choisit $\grave{e}$, conformément à la prononciation : abrègement, allègement, crèmerie (comme crème), évènement (comme avènement), règlementer, règlementation (comme règlement), sècheresse. L'accent aigu (abrégement, crémerie...) ne correspond plus à la prononciation actuelle.

Restent inchangés en raison de leur prononciation «é » en syllabe initiale

- les préfixes dé- et pré- : dégeler, démesuré ; prélever, prémonition, prévenir, etc. ;

- les é à l'initiale de mots : échelon, élever, émeraude, épeler, éperon, etc. ;

- médecin et médecine.

\section{5) Souvent le changement ne touche qu'un accent circonflexe sur $i$ et $u$}

D'une part, quand il n'a plus de fonction par rapport à l'oral ni par rapport à la grammaire de la langue, l'accent circonflexe peut être omis sur les lettres $i$ et $u$, uniquement. Six mots seulement sont touchés sur les mille mots les plus fréquents : boite, couter, bruler, maitre, surement, aout. Pour certaines de leurs formes, il faut ajouter les verbes connaitre, paraitre, reconnaitre, plaire et naitre.

La suppression de l'accent circonflexe sur certains $i$ et $u$ a entrainé de vives réactions parmi les usagers attachés à ce petit «chapeau ». Pourtant, dans les écrits quotidiens, il n'est pas rare de rencontrer : peinture fraiche, chaine hifi, ile, etc.

En faveur du maintien de l'accent circonflexe, on avance que ce signe informe sur le remplacement d'un $s$ disparu. C'est vrai pour quinze des cinquante-six mots avec $\hat{\imath}$ ou $\hat{u}$, ce qui représente à peu près un mot sur quatre. L'accent circonflexe a eu beaucoup d'autres rôles qu'il serait trop long d'exposer ici. Dès lors, on peut créer de nouvelles habitudes visuelles chez les jeunes enfants qui n'auront pas à surcharger inutilement leur mémoire.

D'autre part, lorsque sur $i$ ou $u$, l'accent circonflexe remplit une réelle fonction, celle qui permet d'accéder à la reconnaissance visuelle immédiate d'un mot par rapport à l'écriture d'un autre mot prononcé de la même façon, il est, bien sûr, conservé. Il est maintenu dans deux cas.

$1^{\circ}$ Pour distinguer quelques mots qui sans l'accent seraient homographes : je croîs, elle crût (du verbe croitre) et je crois, elle crut (du verbe croire); payer son dî et manger $d u$ pain ; un fruit mûr et un mur de briques ; à coup sûr et sur le coup ; rompre le jeûne et un jeune garçon; toutefois, certains estiment ce " luxe » inutile, le contexte du mot indiquant clairement de quoi il s'agit. Pour distinguer, dans les verbes dont l'infinitif n'est pas en $-e r$, la $3^{\mathrm{e}}$ personne du subjonctif imparfait (êut, fitt, fût, etc.) et la $3^{\mathrm{e}}$ personne du passé simple (eut, fit, fut, etc.).

$2^{\circ}$ Pour uniformiser les formes du passé simple $\left(1^{\text {re }}\right.$ et $2^{\mathrm{e}}$ personne du pluriel), nous vîmes, vous reçûtes et nous chantâmes, vous chantâtes.

Les noms propres et les adjectifs issus de ces noms gardent leur accent circonflexe : Benoît, l'Île-de-France, Nîmes, l'histoire nîmoise.

XLinguae, Volume 12 Issue 1XL, January 2019, ISSN 1337-8384, eISSN 2453-711X 
Sur les lettres $i$ et $u$, l'accent circonflexe n'a pas de portée phonétique. Comparez: coup et coût, route et croûte, goutte et goûte, tait et plaît, prit et prît, chapitre et épitre. Tandis que sur les lettres $a, e, o$, il joue parfois un rôle en indiquant une différence dans leur prononciation. Aussi l'accent circonflexe est-il maintenu sur les voyelles $a, e, o$.

\section{6) Singulier et pluriel de mots empruntés}

Les mots empruntés à d'autres langues, dont le latin, s'écrivent avec des accents conformes aux règles du français et forment régulièrement leur pluriel comme les mots français, avec un $s$ non prononcé. Braséro, diésel, édelweiss, pédigrée, pérestroïka, péso, piéta, pizzéria, révolver, sombréro. Des allégrettos. Des allégros. Les barmans. Des matchs. Deux pénaltys. Des sandwichs. Des scénarios. Deux whiskys.

On choisit comme forme du singulier la forme la plus fréquente, même s'il s'agit d'un pluriel dans la langue d'emprunt du mot. Un confetti, des confettis. Un graffiti, des graffitis. Un ravioli, des raviolis. Un zakouski, des zakouskis.

Comme en français, les mots terminés par $-s,-x,-z$ ne prennent pas de marque supplémentaire pour former leur pluriel. Des boss. Des box. Des kibboutz.

De plus, les noms composés étrangers sont soudés (cf. supra, A 2). Des cowboys. Des hotdogs. Des exvotos. Des ossobucos. Deux weekends. Cette règle vise à encourager l'intégration des mots étrangers au français.

Mots d'origine latine intégrés au vocabulaire français. Artéfact, critérium, déléatur, désidérata, égo, exéat, facsimilé, mémento, mémorandum, placébo, référendum, sénior, vadémécum, véto. Des désidératas. Les médias. Des satisfécits.

Toutefois, les mots ayant conservé une valeur de citation restent invariables parce que ce sont des extraits de phrases empruntés à une autre langue, en l'occurrence au latin. Des mea culpa. Des Ave Maria. Des Te Deum. Ce sont des noms de prières ou de chants religieux désignés par leur ou leurs premiers mots latins. On les écrit généralement en italique. Des Pater comme : des Notre Père. Le Credo. Des confiteor. Des gloria. Des kyrie ou kyrié. Des magnificat. Des requiem.

7) Le participe passé laissé suivi d'un infinitif ne varie pas. En effet, il joue devant l'infinitif un rôle d'auxiliaire analogue à celui du participe passé fait suivi d'un infinitif, qui est toujours invariable dans ce cas. Elle s'est laissé mourir (comme : elle s'est fait maigrir). Elle s'est laissé séduire (inchangé). Les oiseaux que tu as laissé s'envoler (comme: que tu as fait s'envoler). Littré reconnaissait déjà cette orthographe.

\section{Bilan}

Les rectifications ne constituent pas une révolution, ni une grande réforme, ni une remise en cause de tout le système graphique du français. Les linguistes parlent d'une réformette, affaiblie par des exceptions (parfois regrettables). Positivement, ces aménagements sont utiles parce que les rectifications de 1990 correspondent en grande partie à l'évolution naturelle de la prononciation et des graphies.

Elles touchent 2400 mots, dont certains sont très rares dans l'usage (embattre, écrit avec deux $t$, comme battre; la conjugaison d'agneler). Lorsqu'on les applique toutes, moins d'un mot par page est modifié. Et souvent, il ne s'agit que d'un accent. Sur les 2 400, 800 appartiennent à l'usage commun et 400, au milieu scolaire.

De plus en plus de personnes utilisent spontanément l'orthographe nouvelle. Elle se répand sans être enseignée. Comme elle va dans le sens d'une rationalisation du système, on eût pu souhaiter une mise en ordre plus ample. 


\section{Des variantes recommandées}

Écrire en orthographe reste la norme. L'orthographe lexicale et l'orthographe grammaticale, qui manifeste une maitrise de la syntaxe, visent à faciliter la communication écrite et à contribuer à « la propreté du style » (Madame de Sévigné). Désormais, dans les textes, il convient d'accepter les rectifications de 1990. Cela ne signifie pas être obligé de les appliquer. Les graphies rectifiées ne sont pas imposées. Les usagers peuvent continuer, aussi longtemps qu'ils le souhaitent, à se servir des formes traditionnelles, fussent-elles absurdes ou désordonnées. Il est vrai que ce sont les formes qu'ils ont apprises, parfois péniblement. Toutefois, convient-il d'imposer aux jeunes ces absurdités et ce désordre ?

Les rectifications de 1990 sont, en fait, des variantes orthographiques recommandées par l'Académie française. Aux 3000 variantes anciennes, lexicales du type clef ou clé - et grammaticales - du type il payera ou paiera -, qui se trouvent dans les dictionnaires, s'ajoutent 2400 variantes nouvelles. La nouvelle orthographe se résume à 2400 variantes. Pour chaque variante, aucune des deux graphies, ni l'ancienne, ni la nouvelle, ne peut être tenue pour fautive.

Puisque les graphies anciennes restent valables, les deux orthographes auront à coexister pendant un temps indéterminé, jusqu'à ce que les nouvelles dominent dans l'usage. L'inquiétude n'est donc pas de mise. La langue elle-même n'est pas touchée. Seule son orthographe, son vêtement, s'adapte à l'usage de notre époque.

Les correcteurs orthographiques - comme Antidote (Druide) et Cordial (Synapse) - et les dictionnaires - comme le Dictionnaire Hachette (depuis 2005) et le Petit Larousse illustré (depuis 2012) - mentionnent toutes les rectifications.

Le Conseil international de la langue française (CILF) et la Fédération internationale des professeurs de français (F.I.P.F.) en recommandent l'emploi à tous les enseignants. En effet, il est « plus que temps de redonner à l'orthographe sa place, toute sa place mais rien que sa place, en cessant d'en faire cette institution immuable à laquelle on ne peut toucher sans courir le risque de voir s'effondrer notre langue et nos valeurs culturelles. L'orthographe doit redevenir ce qu'elle n'aurait jamais dû cesser d'être, un objet pratique au service d'une communication écrite accessible au plus grand nombre. » (J.-P. Jaffré, 2009.)

\section{Bibliographic references}

DICTIONNAIRE DE L'ACADÉMIE FRANÇAISE. Paris : Imprimerie Nationale Fayard; $9^{\mathrm{e}}$ édition, 1986, 2000 et 2011, 3 vol., et fascicules parus jusqu'à sabéisme, 28 décembre 2017.

DUPRIEZ, D. 2009 et 2013. La nouvelle orthographe en pratique. Bruxelles : De Boeck-Duculot ; 2 tomes, 240 p. et 144 p.

FAIRON, C. - SIMON, A.-C. 2018. Le Petit Bon usage de la langue française. Grammaire, d'après l'œuvre de Maurice Grevisse. Louvain-la-Neuve : De Boeck Supérieur, $576 \mathrm{p}$.

GOOSSE, A. 1991. La « nouvelle » orthographe. Exposé et commentaires. Louvainla-Neuve : Duculot, 136 p.

GREVISSE, M. - GOOSSE, A. 2016. Le bon usage. Louvain-la-Neuve : De Boeck Supérieur ; $16^{\mathrm{e}}$ édition, $1750 \mathrm{p}$.

GREVISSE, M. - LENOBLE-PINSON, M. 2009. Le français correct. Guide pratique des difficultés. Bruxelles : De Boeck; 6 édition, 512 p.

XLinguae, Volume 12 Issue 1XL, January 2019, ISSN 1337-8384, eISSN 2453-711X 
JAFFRE, J.-P. 2009 «L'orthographe du français. Une histoire, des problèmes. » Langage et pratiques, $\mathrm{n}^{\circ} 43$, juin, 10-18. Accessible en ligne.

LENOBLE-PINSON, M. 2019. Dire et écrire le droit en français correct. Au plaisir des gens de robe. Bruxelles : Éditions Bruylant; $2^{\mathrm{e}}$ édition, $850 \mathrm{p}$.

LENOBLE-PINSON, M. 2017. Écrire sans faute. Dictées lues, commentées et corrigées. Louvain-la-Neuve : De Boeck Supérieur ; $3^{\mathrm{e}}$ édition, 205 p.

Deux sites

www.orthographe-recommandee.info

www.renouvo.org

Words: 4865

Characters : 31683 (17,60 standard pages)

Michèle Lenoble-Pinson

University Saint-Louis, Brussels

Boulevard du Jardin botanique 43, 1000 Brussels

Belgium

michele.lenoble@skynet.be 